\title{
FATORES ASSOCIADOS À QUALIDADE DE VIDA DE CUIDADORES FAMILIARES DE IDOSOS
}

\author{
FACTORS ASSOCIATED WITH THE QUALITY OF LIFE OF \\ FAMILY CAREGIVERS OF ELDERLY PEOPLE
}

\section{FACTORES ASOCIADOS A LA CALIDAD DE VIDA DE CUIDADORES FAMILIARES DE ANCIANOS}

\author{
Karla Ferraz dos Anjos* \\ Rita Narriman Silva de Oliveira Boery ${ }^{* *}$ \\ VANESSA CRUZ SANTOS ${ }^{* * *}$ \\ EdUARdo Nagib BoERY ${ }^{* * * *}$ \\ JaINE KaRENY DA SILVA ${ }^{* * * * *}$ \\ Darci De Oliveira SANTA Rosa ${ }^{* * * * *}$
}

\begin{abstract}
RESUMO
Objetivo: Identificar os fatores associados à qualidade de vida de cuidadores familiares de idosos residentes em domicílio. Material e método: Estudo transversal e censitário realizado com 71 cuidadores de idosos, residentes em um município do interior do Estado da Bahia, Brasil. Os dados foram coletados a partir dos instrumentos de Katz para a avaliação da independência funcional, questionário sociodemográfico e de saúde, escala de Zarit para verificação da sobrecarga e WHOQOL-bref para avaliação da qualidade de vida. Realizou-se análise estatística descritiva e inferencial, de correlação e regressão linear múltipla. Resultados: Maior parte da amostra foi de cuidadoras de meia-idade ou idosas, com reduzida renda mensal individual, com presença de doenças e cuidavam de idosos dependentes. Entre os fatores associados à qualidade de vida dessas pessoas estão a idade, a renda e a sobrecarga do cuidador; o grau de dependência e o gênero do idoso, assim como a quantidade de pessoas que residem no mesmo domicílio. Conclusão: Os fatores que mais impactaram na qualidade de vida do cuidador foram a sobrecarga e o grau de dependência do idoso. Estratégias de fortalecimento do apoio social e orientações sobre o cuidado ao idoso se fazem indispensáveis e podem minimizar a sobrecarga e favorecer a melhoria da qualidade de vida do cuidador.
\end{abstract}

Palavras chave: Qualidade de vida; Cuidadores; Família; Idoso; Enfermagem.

\footnotetext{
* Enfermeira, Doutora, Programa de Pós-graduação em Enfermagem, Universidade Federal da Bahia (PPGENF/UFBA). Bahia, Brasil. Email: karla.ferraz@hotmail.com. Autor de correspondência.

${ }^{* *}$ Enfermeira, Professora Pós-Doutora, Graduação/Programa de Pós-graduação em Enfermagem e Saúde, Universidade Estadual do Sudoeste da Bahia (PPGES/UESB). Bahia, Brasil. Email: rboery@gmail.com

${ }^{* * *}$ Enfermeira, Doutoranda, Instituto de Saúde Coletiva, Universidade Federal da Bahia (ISC/UFBA). Bahia, Brasil. Email: vanessacrus@hotmail.com

**** Enfermeiro, Professor Doutor, Graduação/Programa de Pós-graduação em Enfermagem e Saúde, Universidade Estadual do Sudoeste da Bahia (PPGES/UESB). Bahia, Brasil. Email: eboery@hotmail.com

***** Enfermeira, Doutoranda, Programa de Pós-graduação em Enfermagem e Saúde, Universidade Estadual do Sudoeste da Bahia (PPGES/UESB). Bahia, Brasil. Email: jainekareny@yahoo.com.br

${ }^{* * * * *}$ Enfermeira, Professora Pós-Doutora, Graduação/Programa de Pós-graduação em Enfermagem, Universidade Federal da Bahia (PPGENF/UFBA). Bahia, Brasil. Email: darcisantarosa@gmail.com
} 


\begin{abstract}
Objective: To identify factors associated with the quality of life of family caregivers of elderly people living at home. Material and method: Cross-sectional and census study carried out with 71 caregivers of elderly residents in an inland city of the state of Bahia, Brazil. Data were collected using the Katz instruments for the assessment of functional independence, socio-demographic and health survey, Zarit overload scale and WHOQOLBREF to measure quality of life. Descriptive and inferential statistical analysis, correlation and multiple linear regression were applied. Results: Most of the sample corresponded to middle-aged or elderly female caregivers with reduced individual monthly income, affected by disease and responsible for dependent elderly people. Among the factors associated with quality of life of these people are age, income and caregiver overload; the degree of dependence and gender of the elderly person, as well as the number of people residing in the same household. Conclusion: The factors that had a bigger impact on the caregiver's quality of life were overload and the degree of dependence of the elderly person. Strategies to strengthen social support and guidance on elderly care are essential and can help reduce overload and improve caregiver's quality of life.
\end{abstract}

Key words: Quality of life; caregivers; family and relatives; elderly people; nursing.

\title{
RESUMEN
}

Objetivo: Identificar los factores asociados a la calidad de vida de cuidadores familiares de ancianos residentes en domicilio. Material y método: Estudio transversal y censal efectuado con 71 cuidadores de ancianos, residentes en una ciudad del interior del Estado de Bahía, Brasil. Los datos se recopilaron a partir de los instrumentos de Katz para la evaluación de la independencia funcional, cuestionario sociodemográfico y de salud, escala de Zarit para verificación de la sobrecarga y WHOQOL-bref para evaluación de la calidad de vida. Se realizó análisis estadístico descriptivo e inferencial, de correlación y regresión lineal múltiple. Resultados: La mayor proporción de la muestra fue de cuidadoras de mediana edad o ancianas, con reducida renta mensual individual, con presencia de enfermedades y que cuidaban de ancianos dependientes. Entre los factores asociados a la calidad de vida de esas personas están la edad, la renta y la sobrecarga del cuidador; el grado de dependencia y el género del anciano, así como la cantidad de personas que residen en el mismo hogar. Conclusión: Los factores que más impactaron en la calidad de vida del cuidador fueron la sobrecarga y el grado de dependencia del anciano. Estrategias de reforzamiento al apoyo social y orientaciones sobre el cuidado al anciano se hacen indispensables y pueden mitigar la sobrecarga y favorecer la mejora de la calidad de vida del cuidador.

Palabras clave: Calidad de vida; Cuidadores; Familia; Anciano; Enfermería.

Fecha recepción: 17/07/18 Fecha aceptación: 09/10/18

\section{INTRODUÇÃO}

Paralelo à transição demográfica ocorrem mudanças no perfil epidemiológico da população e aumento de doenças crônicas que, em algumas situações, comprometem a autonomia do idoso $^{(1)}$. Isso demanda cuidados permanentes da família e assistência contínua e específica da equipe de saúde. Embora a dependência não seja inerente ao processo de envelhecimento, esta tem comprometido a autonomia de inúmeros idosos.

No Brasil, as principais causas do aumento das taxas de idosos com incapacidades são a frequência das doenças crônicas e a longevidade. Desse modo, a prevenção das doenças crônicas e degenerativas, a assistência à saúde dos idosos dependentes e o apoio aos cuidadores familiares representam desafios para o sistema de saúde. No país, o idoso sem independência e autonomia faz parte de parcela oculta da opinião pública, e isto se deve, em parte, à manutenção deste nos do- 
micílios ou em instituições de longa permanência para idosos, dificultando a visibilidade e, por conseguinte, a preocupação política de proteção social $^{(2)}$.

Estudo demonstra que, com a perda da independência e da autonomia do idoso no domicílio, alguém assume a função de cuidador, pessoa que se responsabiliza em desenvolver as tarefas cotidianas as quais a pessoa dependente não mais consegue realizar sozinha. Assim, o envelhecimento, sobretudo com dependência, e o cuidador exigem novas maneiras de assistência por parte dos profissionais de saúde e novos enfoques por parte das políticas públicas de saúde ${ }^{(2)}$.

Frente à dependência do idoso, na maioria das vezes, a família se responsabiliza pelo cuidado em virtude das relações, valores construídos e atribuídos, assim como das representações que possuem de atitude e preocupação ${ }^{(3)}$. Ressalta-se que familiares também estão cuidando de idosos sadios, considerados independentes em termos funcionais e/ou cognitivos, que, no entanto, necessitam de cuidados e apoio no desenvolvimento de suas atividades cotidianas, contribuindo para o crescimento de cuidadores informais. Essa demanda precisa ser considerada e investigada para que ações de promoção de um envelhecimento ativo e prevenção dessas situações sejam implementadas junto aos idosos e suas famílias.

Os cuidados aos idosos prestados por familiares variam a depender da necessidade de cada pessoa. Idosos dependentes em atividades básicas ou instrumentais demandam maior tempo de cuidados diários e diretos. Aos idosos independentes, a exigência de cuidados ocorre em menor proporção, de forma pontual e esporádica. Estudo $^{(4)}$ identificou que idosos considerados independentes apresentam dificuldades para executar atividades diárias como realizar os trabalhos domésticos, cuidar das finanças, fazer compras, viajar, usar o telefone e automedicar-se. E idosos dependentes sentem dificuldades para realizar o autocuidado como banho, alimentação, vestuário, e controle de micção ${ }^{(4)}$. Neste momento, surge a necessidade do cuidador para auxiliar ou mesmo fazer pelos idosos as atividades que sozinhos não mais conseguem desenvolver.
O cuidador familiar ao assumir o cuidado do idoso, comumente, o faz de maneira ininterrupta, não se permitindo, às vezes, ter tempo para $\mathrm{o}$ atendimento de suas atividades pessoais e/ou profissionais. Assim, verifica-se alteração do estilo de vida do cuidador em função das necessidades do idoso. Atividades de recreação e convívio social normalmente acabam sendo postergadas em decorrência das demandas de cuidado a depender das exigências do idoso, especialmente quando este necessita da presença constante desse membro da família ${ }^{(5)}$. Nessa perspectiva, surgem implicações na saúde e na qualidade de vida (QV) dos cuidadores.

Cabe destacar que a responsabilidade do cuidador vai além da vontade de querer realizar o cuidado do seu familiar. Esta envolve conhecimentos, competências, habilidades, iniciativas para ações de promoção, tratamento e recuperação da saúde do idoso. Tal responsabilidade é considerada pelos cuidadores como "difícil" por exigir paciência, amor e renúncia de seu projeto de vida. Além disso, contribui para que estes vivenciem a sobrecarga física, emocional e social ${ }^{(6)}$, assim podem ter implicação em sua QV, pela influência de fatores vivenciados cotidianamente. Talvez, o que torna a tarefa de cuidado mais "difícil" é o fato de esta exigir conhecimentos específicos que o cuidador informal, muitas vezes, não possui.

Constata-se que o envelhecimento populacional e a evidente demanda de cuidadores tornam as pessoas vulneráveis à sobrecarga e ao comprometimento da $\mathrm{QV}^{(7)}$, esta, conforme o grupo World Health Organization Quality of Life Assessment é definida como a percepção que o indivíduo tem sobre sua posição na vida, no contexto da cultura e do sistema de valores em que vive e em relação aos seus objetivos, expectativas, padrões e preocupações ${ }^{(8)}$.

Entre os cuidadores, à medida que aumenta a sobrecarga, ocorre piora da $\mathrm{QV}^{(7)}$. Isso pode estar relacionado às atividades de cuidado do idoso dependente ou não, as quais se somam as atividades cotidianas do cuidador, ou seja, à (re)adaptação do cuidador no domicílio cuidando de uma pessoa que necessita de ajuda/apoio continuado. 
A responsabilidade atribuída ao cuidador familiar, muitas vezes, sem orientação adequada, suporte de instituições de saúde e alteração da rotina no cuidado têm implicado na sua $\mathrm{QV}^{(9)}$. Aditivamente, tem-se o déficit ou inexistência de apoio da sociedade para a realização dos cuidados ao idoso, os quais se somam as atividades diárias que o cuidador tem de exercer para manter sua própria subsistência.

Os cuidadores necessitam ser orientados e preparados para executarem o cuidado apropriado ao idoso no domicílio, serem capazes de avaliar as necessidades, potencialidades e limites da pessoa cuidada, assim como gerenciar e executar seu autocuidado. Portanto, é fundamental o profissional de saúde incorporar ações educativas aos cuidadores em sua prática assistencial, respeitar e valorizar a participação e autonomia dessas pessoas, assim como possibilitar que seus anseios e suas dúvidas sejam minimizados. Desta maneira, estes cuidadores se sentirão mais seguros em suas ações, o que traz benefício ao cuidador e ao idoso ${ }^{(10)}$.

Formulou-se como hipótese do estudo: a qualidade de vida dos cuidadores familiares de idosos associa-se negativamente com condições socioeconômica e de saúde, sobrecarga de atividades, idade, gênero, grau de dependência do idoso, tempo de cuidado e apoio social. Nesse contexto, o objetivo deste estudo consistiu em identificar os fatores associados à qualidade de vida de cuidadores familiares de idosos residentes em domicílio.

\section{MATERIAL E MÉTODO}

Estudo transversal e censitário realizado em residências, da área de abrangência de duas Estratégias Saúde da Família (ESF) da zona urbana do município de Manoel Vitorino, no período de janeiro a fevereiro de 2013. Este município conta com quatro ESF (duas na zona urbana e duas na zona rural), está localizado na região Sudoeste da Bahia, Brasil, e sua população é de 14.387 indivíduos, concentrando-se cerca de 7.359 pessoas na área urbana e, destes, sendo 871 idosos $^{(11)}$.

No contexto geral, a população do município de Manoel Vitorino apresenta características de desigualdade social e de pobreza, pois predominantemente é composta por indivíduos com baixo poder aquisitivo ${ }^{(11)}$. O Índice de Desenvolvimento Humano Municipal (IDHM) é de 0,566 e o Índice de Gini é de 0,51, sendo a renda per capita de R $\$ 226,95$, com $26,47 \%$ de indivíduos extremamente pobres e $46,92 \%$ pobres $^{(12)}$.

Participaram deste estudo 71 cuidadores familiares de idosos dependentes. Para tanto, fez-se busca nas Fichas A, de cada uma das microáreas das ESF para listar os idosos cadastrados e atendidos pelas equipes de saúde. Posteriormente, após visita no domicílio identificou-se todos os idosos dependentes e independentes que tinham cuidadores. Considerou-se como familiar, as pessoas com vínculos, nem sempre caracterizadas por laços consanguíneos, mas também de coabitação. E como cuidador familiar a pessoa da família que cuidava do idoso no domicílio.

Os critérios de inclusão adotados foram: ser o cuidador familiar principal do idoso; coabitar o mesmo domicílio; idade igual ou superior a 18 anos; estar cadastrado em uma das ESF. Os indivíduos que após três visitas em dias e horários distintos não foram encontrados para a entrevista foram excluídos. Ressalta-se que todos os cuidadores identificados participaram do estudo, não havendo perda e nem recusa.

$\mathrm{Na}$ visita domiciliar foi aplicada a Escala de Independência em Atividades da Vida Diária (EIAVD), ou escala de Katz, para avaliar a independência funcional do idoso cuidado em seis áreas de funcionamento (banho, vestir, sanitário, transferência, continência e alimentação), classificando-o como independente ou dependente em uma, duas, três, quatro, cinco ou seis funções (totalmente dependente), visto que o escore varia de zero a seis pontos, sendo um ponto atribuído para cada resposta "Sim”, a depender da descrição que se aplica a resposta ${ }^{(13)}$. Este instrumento foi respondido pelos cuidadores.

Em seguida, aplicou-se um questionário, elaborado pelos autores, para a verificação das características sociodemográficas e de saúde do 
cuidador e do idoso cuidado, composto dos seguintes itens: 1) Cuidador: gênero, idade, raça/ cor, estado civil, escolaridade, horas diárias dedicadas ao cuidado, tempo (ano) que cuida, renda, parentesco, trabalho, presença de doenças, uso de medicação, apoio social, orientações sobre cuidados aos idosos e quantidade de pessoas que residem no domicílio e; 2) Idoso cuidado: gênero, idade, problemas de saúde, independência e dependência.

A sobrecarga do cuidador familiar foi verificada a partir da escala Zarit Burden Interview a qual consiste em 22 questões que avaliam condição de saúde, bem-estar psicológico, finanças e vida social. Esta escala é do tipo Likert, graduada de 0 a 4 e pontuada conforme a presença e intensidade de uma afirmativa ( $0=$ nunca, $1=$ raramente, 2 =algumas vezes, $3=$ frequentemente e $4=$ sempre). Para a última questão há exceção, visto que o entrevistado é questionado se está se sentindo sobrecarregado com a responsabilidade atribuída à função de cuidador, sendo as respostas: $0=$ nem um pouco, $1=$ um pouco, $2=$ moderadamente, $3=$ muito, 4 = extremamente. $\mathrm{O}$ escore da escala varia de 0 a 88 , sendo a sua proporção de quanto maior a pontuação, maior é a sobrecarga ${ }^{(14)}$.

A QV dos cuidadores foi avaliada por meio do questionário World Health Organization Quality of Life (WHOQOL-bref), no qual constam 26 questões - duas gerais (QV geral e satisfação com a saúde) e as 24 demais representam as facetas dos domínios físico, psicológico, relações sociais e meio ambiente ${ }^{(15)}$. As duas questões gerais foram avaliadas juntas para gerar o Índice Geral de Qualidade de Vida (IGQV), independente dos escores dos domínios.

Para o processamento dos dados utilizou-se o software Statistical Package for Social Science (SPSS) versão 18.0. Optou-se pela análise estatística descritiva e inferencial com a utilização da média, mediana e desvio padrão (DP); a correlação de Spearman entre as variáveis de desfecho: IGQV e os domínios do WHOQOL-bref (físico, psicológico, relações sociais e meio ambiente) e as variáveis de exposição (idade do idoso, gênero do idoso, idade do cuidador, renda do cuidador, número de pessoas que reside no domicílio, tem- po como cuidador, horas dedicadas ao cuidado, sobrecarga e grau de dependência), assim como o modelo de regressão linear múltipla final (stepwise forward), tendo como variável de desfecho o escore do IGQV por considerá-lo um indicador abrangente ao objetivo deste estudo, as variáveis de exposição. O nível de significância adotado foi de $5 \%$.

O estudo foi aprovado pelo Comitê de Ética em Pesquisa da Universidade Estadual do Sudoeste da Bahia, campus de Jequié, Bahia, Brasil, sob o Parecer Consubstanciado n ${ }^{\circ}$. 128.580/2012 e CAAE: 08643612.6.0000.0055. Todos os cuidadores assinaram o Termo de Consentimento Livre e Esclarecido após convite e aceite de participação do estudo.

\section{RESULTADOS}

Apresenta-se, inicialmente, os dados referentes aos idosos cuidados: constatou-se predomínio de mulheres $(67,6 \%)$ com idade média de 79,5 anos (DP 10,6). Os problemas de saúde dos idosos mais citados pelos cuidadores foram: hipertensão arterial (49,2\%), diabetes mellitus $(15,4 \%)$ e sequelas de acidente vascular cerebral (14\%). Dos idosos, $81,8 \%$ foram classificados como dependentes de cuidados e, destes, $38,1 \%$ eram dependentes de uma a três funções, $43,7 \%$ de quatro a seis funções e 18,2\% eram totalmente dependentes, sendo a mediana da escala de Katz, utilizada para realizar esta avaliação de 4,0 $( \pm 2,1)$.

A população foi constituída de 71 cuidadores de idosos, dos quais $60(85,9 \%)$ eram do gênero feminino, $44(62,2 \%)$ casados, $42(59,2 \%)$ com filhos, 53 (74,7\%) declararam ser negros (pardos e pretos), $56(78,9 \%)$ estavam desempregados, 23 $(32,4 \%)$ não possuíam renda mensal individual e, entre os que tinham renda mensal, a mediana foi de 172,00 reais. A média de idade foi de 47 anos (DP 16,1), sendo 18 (25,4\%) cuidadores idosos. Em relação à escolaridade, 33 (46,9\%) tinham ensino fundamental incompleto ou completo e 19 (25,9\%) eram analfabetos. Quanto ao tempo (anos/horas) de duração da atividade cui- 
dadora, $36(51,0 \%)$ dedicavam superior a cinco (média de 21,1 horas diárias), conforme expresso anos e $64(90,2 \%)$ cuidavam de maneira integral na Tabela 1.

Tabela 1. Distribuição das características sociodemográficas dos cuidadores familiares de idosos $(\mathrm{n}=71)$. Manoel Vitorino, Bahia, Brasil, 2013.

\begin{tabular}{|c|c|}
\hline Variável & $\begin{array}{c}\text { Cuidadores familiares } \\
\qquad(\%)\end{array}$ \\
\hline \multicolumn{2}{|l|}{ Gênero } \\
\hline Feminino & 85,9 \\
\hline Masculino & 14,1 \\
\hline \multicolumn{2}{|l|}{ Idade } \\
\hline Média & 47 \\
\hline Desvio padrão & 16,1 \\
\hline Mínimo & 18 \\
\hline Máximo & 80 \\
\hline \multicolumn{2}{|l|}{ Raça/cor } \\
\hline Branca & 25,3 \\
\hline Parda & 66,2 \\
\hline Preta & 8,5 \\
\hline \multicolumn{2}{|l|}{ Estado civil } \\
\hline Casado / União consensual & 62,2 \\
\hline Solteiro/Divorciado & 33,8 \\
\hline Viúvo & 4,2 \\
\hline \multicolumn{2}{|l|}{ Escolaridade } \\
\hline Analfabeto & 25,9 \\
\hline Ensino fundamental incompleto & 39,9 \\
\hline Ensino fundamental completo & 7 \\
\hline Ensino médio incompleto/completo & 23 \\
\hline Superior incompleto/completo ou mais & 4,2 \\
\hline \multicolumn{2}{|l|}{ Horas por dia dedicadas ao cuidado } \\
\hline Até 8 & 9,8 \\
\hline Superior a 8 & 90,2 \\
\hline \multicolumn{2}{|l|}{ Tempo (em anos) que cuida } \\
\hline Menor que 1 & 7 \\
\hline De 1 a 5 & 42 \\
\hline Superior a 5 anos & 51 \\
\hline \multicolumn{2}{|l|}{ Renda mensal $(\mathrm{SM})^{\star}$} \\
\hline Sem renda & 32,4 \\
\hline Até meio SM (310,00 reais) & 28,1 \\
\hline Entre meio e $1 \mathrm{SM}$ (320,00 a 620,00 reais) & 31,1 \\
\hline Maior que $1 \mathrm{SM}$ & 8,4 \\
\hline \multicolumn{2}{|l|}{ Parentesco com relação ao idoso } \\
\hline Filho (a) & 59,2 \\
\hline Cônjuge & 19,7 \\
\hline
\end{tabular}


Continuação Tabela 1.

Neta/ Sobrinha

Outros (Irmã/Nora)

Trabalho

Exerce atividade remunerada

Desempregado/ Dona de casa

Nunca exerceu trabalho remunerado

Presença de doença do cuidador

Uso de medicação diária do cuidador

Recebe ajuda informal

Não recebe ajuda formal

Pessoas que residem no domicílio (Média)

(*) Salário Mínimo $(\mathrm{SM})=\mathrm{R} \$ 620,00$ reais

Todos os cuidadores residiam no mesmo domicílio que o idoso, coabitando em média 3,6 pessoas (DP 2,2), como demonstra a Tabela 1. Concernente à rede de apoio social, 42 (59,2\%) recebiam ajuda de familiares e/ou amigos para realizar o cuidado ao idoso. Os cuidadores (100\%) não mencionaram ajuda/apoio de profissionais de saúde no cuidado ao idoso. Em relação à situação de saúde do cuidador, 61 (85,9\%) referiram-se à presença de uma doença ou comorbidades, destas, as mais autodeclaradas foram: problemas na coluna $(59,1 \%)$, hipertensão arterial $(36,6 \%)$ e varizes $(36,6 \%)$. Entre os cuidadores, $33(46,5 \%)$ faziam uso de medicações diárias.

Como forma de averiguar se os cuidadores familiares tinham sido informados por profissionais de saúde e/ou comunidade sobre como cuidar do idoso no domicílio, foi questionado se haviam participado de curso, reunião e/ou grupo de orientações para ajudá-los nos cuidados. Não houve nenhum cuidador (100\%) que afirmasse ter participado; mencionaram ter aprendido com a prática cotidiana. A escala Zarit de mensuração da sobrecarga variou de 15 a 72 pontos, com média de 43,2 (DP 14,3). No WHOQOL-bref, o escore do IGQV foi de 55,6 pontos, o domínio físico $(56,4)$ apresentou o maior escore e o domínio meio ambiente $(40,8)$ o menor.

A Tabela 2 apresenta os coeficientes de correlação de Spearman entre os domínios do WHOQOL-bref e as variáveis quantitativas a respeito do cuidador e do idoso. Houve correlação significante entre a idade do cuidador, renda do cuidador, número de pessoas que reside no domicílio, tempo como cuidador (anos) e as escalas de sobrecarga e dependência. Evidenciou-se que a escala de Zarit (sobrecarga) obteve correlação negativa significativa com todos os domínios da QV e com o IGQV, assim, quanto menor o escore do índice geral e dos domínios da QV, maior a pontuação da escala de Zarit e, portanto, maior a sobrecarga.

A Tabela 3 mostra o modelo de regressão linear múltipla final (stepwise forward), sendo verificado que o grau de dependência e o gênero do idoso, assim como a sobrecarga de atividades do cuidador, foram variáveis significantes que relacionaram com o IGQV e permaneceram ao final do modelo. Isso evidencia que essas variáveis juntas foram capazes de explicar a pior QV dos cuidadores. 
Tabela 2. Coeficientes de correlação de Spearman $\left(\mathrm{r}_{\mathrm{sp}}\right)$ entre os domínios do WHOQOL-bref e IGQV com as variáveis de exposição. Manoel Vitorino, Bahia, Brasil, 2013.

\begin{tabular}{lccccc}
\hline \multirow{2}{*}{ Variáveis } & & \multicolumn{4}{c}{ Domínios do WHOQOL-bref } \\
\cline { 3 - 6 } & IGQV & Físico & Psicológico & Relações sociais & Meio ambiente \\
\hline \multirow{2}{*}{ Idade do idoso } & $\mathrm{r}_{\mathrm{sp}}$ & $\mathrm{r}_{\mathrm{sp}}$ & $\mathrm{r}_{\mathrm{sp}}$ & $\mathrm{r}_{\mathrm{sp}}$ & $\mathrm{r}_{\mathrm{sp}}$ \\
& {$[\mathrm{p}]$} & {$[\mathrm{p}]$} & {$[\mathrm{p}]$} & {$[\mathrm{p}]$} & {$[\mathrm{p}]$} \\
Gênero do idoso & 0,083 & 0,005 & $-0,141$ & $-0,083$ & 0,213 \\
& {$[0,490]$} & {$[0,968]$} & {$[0,241]$} & {$[0,491]$} & {$[0,074]$} \\
Idade do cuidador & $-0,226$ & $-0,162$ & $-0,034$ & $-0,023$ & $-0,163$ \\
& {$[0,058]$} & {$[0,177]$} & {$[0,778]$} & {$[0,849]$} & {$[0,175]$} \\
Renda do cuidador & $-0,099$ & $-0,346 \dagger$ & $-0,171$ & $-0,3$ & 0,182 \\
No de pessoas que reside no domicílio & $-0,048$ & $-0,061$ & $-0,117$ & $-0,235^{*}$ & $-0,002$ \\
& {$[0,413]$} & {$[0,003]$} & {$[0,153]$} & {$[0,801]$} & {$[0,128]$} \\
Tempo como cuidador & 0,176 & 0,006 & $-0,159$ & 0,086 & $0,257^{*}$ \\
Horas dedicadas ao cuidado & {$[0,143]$} & {$[0,960]$} & {$[0,184]$} & {$[0,476]$} & {$[0,031]$} \\
& $-0,103$ & $-0,125$ & $-0,001$ & $-0,242^{*}$ & 0,092 \\
Sobrecarga (Zarit) & {$[0,394]$} & {$[0,297]$} & {$[0,995]$} & {$[0,042]$} & {$[0,444]$} \\
& $-0,071$ & $-0,196$ & $-0,131$ & $-0,032$ & 0,018 \\
& {$[0,555]$} & {$[0,102]$} & {$[0,276]$} & {$[0,793]$} & {$[0,882]$} \\
& $-0,541 \dagger$ & $-0,575 \dagger$ & $-0,525 \dagger$ & $-0,391 \dagger$ & $-0,360 \dagger$ \\
& {$[<0,001]$} & {$[<0,001]$} & {$[<0,001]$} & {$[0,001]$} & {$[0,002]$} \\
& $-0,183$ & $-0,300^{*}$ & $-0,324 \dagger$ & $-0,342 \dagger$ & $-0,040$ \\
& {$[0,127]$} & {$[0,011]$} & {$[0,006]$} & {$[0,004]$} & {$[0,739]$} \\
\hline
\end{tabular}

$\left[{ }^{*}\right]$ correlação estatisticamente significativa $[\mathrm{p}<0,05]$

$[\dagger]$ correlação estatisticamente significativa $[\mathrm{p}<0,01]$

Tabela 3. Modelo de regressão linear múltiplo final para o Índice Geral de Qualidade de Vida. Manoel Vitorino, Bahia, Brasil, 2013.

\begin{tabular}{lcc}
\hline Variável & Coeficiente de regressão $\beta$ & p-valor \\
Grau de dependência (Katz) & 0,319 & 0,012 \\
Sobrecarga (Zarit) & $-0,606$ & $<0,001$ \\
Gênero do idoso & $-0,213$ & 0,041 \\
\hline
\end{tabular}

$\mathrm{R}^{2}$ ajustado $=0,273 ; \mathrm{p}$ (modelo) $=0,001$ 


\section{DISCUSSÃO}

Os idosos cuidados eram predominantemente mulheres, com idade de 60 a 102 anos e grau elevado de dependência, embora existissem aqueles independentes. Dados semelhantes foram descritos em estudo nacional ${ }^{(16)}$ ao identificar que os idosos com cuidadores são, especialmente, os mais dependentes, assim como internacional ${ }^{(17)}$ que obteve também essa similaridade. Considera-se que o fato de o gênero do idoso ter influenciado na QV do cuidador pode ter relação com o predomínio de mulheres no estudo.

Com relação ao perfil dos cuidadores familiares ser mulheres, casadas, filhas ou cônjugues, apresentar reduzida escolaridade, baixas condições econômicas e idade avançada (25,4\% são cuidadores idosos), tais achados não se diferenciam tanto dos encontrados em outras regiões do Brasil $^{(16,18)}$ ou em outros países como Espanha ${ }^{(19)}$, França, Alemanha e Reino Unido ${ }^{(17)}$ mesmo tratando-se de estudo desenvolvido em município com baixo IMDH.

As baixas condições econômicas dos cuidadores (32,4\% sem renda) evidenciadas neste estudo podem estar associadas a estes terem de abdicar do trabalho remunerado para cuidar do idoso, além do déficit de oportunidades identificado a partir das características do município que apresenta baixo IDHM. O incentivo ao trabalho a ser desenvolvido no próprio domićlilo pode contribuir com a renda dessas pessoas as quais, muitas vezes, não podem realizar trabalho extradoméstico devido ao tempo disponibilizado para o cuidado ao idoso e, em algumas circunstâncias, não ter com quem deixar o mesmo. Este estudo também apontou correlação da renda do cuidador com o domínio meio ambiente da QV.

Enfatizando a prevalência encontrada de mulheres cuidando dos idosos, esta se apresenta como questão de gênero, pois, realizar o cuidado parece ser uma atribuição feminina e atividade solitária, de ampla exigência física e emocional. Entretanto, algumas mulheres demonstram a face de ser cuidadora e vivenciar sua velhice cuidando do outro ${ }^{(20)}$. Essa questão de gênero a qual envolve o cuidado domiciliar mostra que a saúde da mulher precisa ser ainda mais foco de atenção por parte dos profissionais de saúde, uma vez que o cuidar de outra pessoa pode ocasionar implicações às cuidadoras, como a sobrecarga de atividades, o comprometimento da saúde e da QV, principalmente quando já estão em uma idade avançada e não têm apoio social instituído para ajudá-las nos cuidados ao idoso.

O aumento de idosos decorrente do envelhecimento populacional faz com que eleve a possibilidade de, nas famílias e dentro delas, as mulheres de diferentes faixas etárias, mas especificamente as idosas e as de meia-idade, passem a se dedicar ao cuidado do senescente no domicílio. Atualmente, a maior preocupação é direcionada ao idoso dependente em detrimento de seu cuidador que, muitas vezes, também é idoso, situação essa cada vez mais frequente na sociedade. E, quando o cuidador é idoso torna-se mais uma pessoa vulnerável ${ }^{(21)}$. Neste estudo também predominou o cuidador de meia-idade ou idoso. Para tanto, faz-se necessário estratégias de intervenções junto ao cuidador, com peculiaridades próprias do envelhecimento, as quais favoreçam que esse indivíduo seja cuidado e protegido de agravos à saúde.

Ao considerar o tempo de cuidado ao idoso, a maioria dos cuidadores dedicava-se por períodos longos e de forma integral, dados semelhantes descritos em estudo prévio ${ }^{(18)}$. Em outro estudo foi encontrado tempo médio de 10 anos de cuidado. E, em relação às horas, $67,6 \%$ dos cuidadores dedicam-se integralmente ao cuidado com média 8,2 (DP 5,1) horas/dia ${ }^{(22)}$. Esta dedicação em tempo (horas/anos) pode estar relacionada ao cuidador residir no mesmo domicílio com o idoso e ao grau de dependência deste. Isso contribui para a sobrecarga do cuidador, visto que acrescenta às suas atividades as de cuidado do idoso.

Ao destacar o cuidado no domicílio, verificase tendência das políticas de atenção ao idoso defender como o melhor local para ele viver o seu envelhecimento, a favor da manutenção da autonomia/independência, identidade e dignidade. Contudo, a falta de apoio dos serviços sociais 
e de saúde para o autocuidado da família como unidade, principalmente com membros dependentes de cuidado prolongado, torna-se um ônus que ameaça o bem-estar e a QV da família, assim como desequilíbrio em sua saúde e finanças ${ }^{(23)}$. Assim, com o processo de adoecimento das pessoas, a equipe de saúde necessita estar capacitada para desenvolver ações compartilhadas com diversos setores da sociedade e universidade que minimizem o impacto desta nova condição na dinâmica familiar. Neste contexto, a educação em saúde e o apoio às famílias cuidadoras e aos idosos tornam-se fundamentais.

Sendo assim, o processo de cuidar no domicílio não se constitui apenas em atribuição cuidativa, mas vai além, implicando em preparar ou reorganizar o ambiente para poder lidar bem com a situação. Todavia, isso nem sempre pode ser conduzido, pois, em alguns casos, as questões financeiras atuam como fatores determinantes e condicionantes $^{(24)}$. E, tratando-se de pessoas com baixas condições econômicas, como os participantes deste estudo, isso implica, muitas vezes, em ocasionar desgastes emocionais aos cuidadores quando estes não conseguem (re)adaptar o domicílio para prestar o cuidado com qualidade ao seu familiar idoso.

Ademais, à medida que aumenta a debilidade e a dependência do idoso, os encargos do cuidar podem sofrer novas alterações, exigindo maiores esforços para suprir as necessidades que acompanham a diminuição da capacidade funcional do idoso. Assim, como resultado das repercussões do cuidado e estado de vulnerabilidade, ao qual o cuidador se expõe, pode emergir desgastes físico, psicológico e/ou social e, consequentemente, acarretar sobrecarga para quem cuida ${ }^{(25)}$. Nessa perspectiva, verifica-se a necessidade de intervenções, como a de orientações, educação em saúde e capacitação desses cuidadores informais, conforme demandas dos idosos, pois há possibilidade de sobrecarga entre os cuidadores evoluir para graus elevados (como a severa) e comprometer a QV e a saúde dessas pessoas.

Idosos considerados independentes sentem necessidades de apoio de outras pessoas para o acompanhamento aos serviços de saúde, auxílio para tomar remédio, companhia, administração dos interesses financeiros, aquisição de recursos indispensáveis à manutenção da vida, como as sociais, as quais os envolvem em convívio com pessoas de outras gerações, como forma de socialização. Essas demandas também são identificadas entre os idosos dependentes, acrescidas de outras, como as de cuidado individual, a depender da área de funcionamento conforme escala de $\mathrm{Katz}^{(13)}$, como ajuda para banho, vestir, sanitário, transferência, continência e alimentação.

Estudo demonstra que as variáveis independentes relacionadas com à $\mathrm{QV}$ foram a escala de sobrecarga, a presença de companheiro e idade do cuidador. E os cuidadores que referiram maior sobrecarga avaliaram menos satisfatoriamente sua QV, especialmente quanto à dor, capacidade funcional, aspectos físicos e emocionais ${ }^{(26)}$. Neste estudo, mesmo com variáveis diferentes, esses dados se assemelham em parte com a mesma análise feita e evidenciou correlação do IGQV com o grau de dependência, a escala de Zarit e o gênero do idoso.

Em um estudo foi visto que a maioria (57,3\%) dos cuidadores relatou sobrecarga moderada e o gênero do cuidador associou-se significamente com a sobrecarga, sendo que os homens cuidadores apresentaram maiores chances de menor sobrecarga do que as mulheres, sobretudo, quando o idoso era independente. Também demonstrouse o quanto mais o idoso é independente, maior é a chance de o cuidador ter menor sobrecarga, quando comparados aos cuidadores de idosos com dependência completa ou parcial ${ }^{(25)}$. Logo, torna-se relevante estimular a participação do homem como cuidador de idoso e não somente a mulher, isso porque no domicílio ela tem várias outras atribuições para realizar, assim como ter de conciliar com o cuidado de outros membros da família, como filhos, netos e sobrinhos.

Salienta-se que a cuidadora pode estar sendo responsabilizada por outras atividades, não somente as dedicadas ao idoso, pois, culturalmente, a mulher ainda assume cuidados relacionados ao contexto familiar, acrescidos de sua inserção no mercado de trabalho ${ }^{(25)}$. Além disso, têm-se as condições estruturais as quais envolvem ambien- 
te e finanças e também contribuem para a sobrecarga. Tudo isso indica que a QV do cuidador fica comprometida ${ }^{(24)}$. Essas atividades somadas ao cuidado podem tornar fator associado à saúde do cuidador, especialmente quando este não tem apoio social disponível.

Neste estudo, o apoio social informal é presente para 59,2\% dos cuidadores e apoio formal inexistente. Resultados similares ${ }^{(22)}$ foram encontrados em outro estudo. Destarte, os achados deste estudo tornam-se preocupantes diante dos benefícios que o apoio social pode trazer para o cuidador e idoso e influenciar positivamente na QV do binômio. Logo, é preciso estimular familiares e comunidade, assim como sensibilizar profissionais de saúde a apoiarem os cuidadores.

Vários são os cuidadores familiares de idosos apresentando alguma doença e fazendo uso de medicações. Verifica-se que os problemas de saúde influenciaram negativamente na QV dos cuidadores, especialmente nos domínios físico e psicológico $^{(18)}$. Esses dados convergem aos deste estudo, visto $85,9 \%$ dos cuidadores apresentam alguma doença ou comorbidade e 46,5\% fazem uso de medicações diárias, o que, por sua vez, pode influenciar na QV dessas pessoas.

Foi evidenciado que entre os cuidadores houve alguma mudança em sua vida após a assunção da responsabilidade pelo cuidado, sendo dito por $50 \%$ que ter deixado de trabalhar extradomicílio ${ }^{(27)}$. Embora cuidar possa ser sofrido, a evolução da doença e a aceitação da nova realidade podem conduzir para modificações na vida das pessoas. Alguns cuidadores relatam ter passado por várias etapas e que atualmente são outras pessoas $^{(28)}$. Neste estudo, as mudanças ocorridas na vida do cuidador após a assunção do cuidado ao idoso podem ter relação com o cuidado integral (média de 21,1 horas diárias) e isso impactar na sobrecarga e QV dessas pessoas, particularmente, no domínio relações sociais, visto que vários cuidadores deixam de sair de casa por conta das demandas de cuidado ao idoso.

Considera-se que dentre os fatores que podem influenciar negativamente a QV dos cuidadores estão a doença e grau de dependência do idoso, idade do cuidador e do idoso, sobrecarga de ati- vidades e déficit de apoio social ${ }^{(18)}$. Em relação ao apoio social de familiares, este pode contribuir de maneira positiva na QV dos cuidadores e estar relacionado ao número de membros que reside no mesmo domicílio. Entretanto, a depender da família, quanto maior o quantitativo de pessoas convivendo juntas pode contribuir para maior sobrecarga no cuidador, devido aumentar a demanda de atividades para esse indivíduo. Isso foi visto na redução da QV dos cuidadores no domínio relações sociais quando associado ao número de pessoas que reside no mesmo domicílio.

Também, fatores como depressão, má qualidade do sono, tipo de demência do idoso, apoio e suporte social, acesso aos serviços de saúde, atividades de lazer, problemas de saúde pré-existentes, capacitação do cuidador e religiosidade têm influenciado na QV do cuidador familiar. E estratégias como o suporte social, psicológico e educacional têm impactado positivamente na QV desses. O enfermeiro ao estabelecer vínculo terapêutico com as famílias cuidadoras pode identificar suas necessidades, para, então, traçar ações que proporcionem organização e sistematização do cuidado, educação em saúde e suporte social para essas pessoas superassem dificuldades inerentes ao cuidado do idoso dependente ${ }^{(29)}$. Apontamos também que o grau de dependência do idoso influenciou na QV dos cuidadores nos domínios físico, psicológico e relações sociais do WHOQOL-bref.

Em estudo foi evidenciado que no WHOQOL-bref os domínios físico e relações sociais apresentaram os menores escores, enquanto o domínio meio ambiente e psicológico apresentaram os maiores ${ }^{(30)}$. Em outro de natureza diagnóstico-avaliativa, mostrou-se que a QV dos cuidadores no IGQV foi de 62,4, com pontuação mais baixa nos domínios psicológico e meio ambiente $^{(23)}$. Esses resultados mostram que estes domínios, especialmente o meio ambiente podem sofrer variação e influenciar positiva ou negativamente na QV das pessoas, sendo necessários mais estudos para melhor compreensão dos fatores relacionados. Neste sentido, a equipe de saúde necessita considerar uma maior avaliação do ambiente no planejamento de suas ações de 
cuidado, sobretudo, quando for realizar visita e/ ou assistência domiciliar.

Estudo constata que o escore encontrado pelo grau de dependência correlacionou-se negativamente com a maioria dos domínios do WHOQOL-bref, exceto meio ambiente. Além disso, houve associação negativa significativa do domínio relações sociais com o tempo do cuidador que mora com o idoso, indicando que quanto maior o tempo de convivência, menor a percepção de QV neste domínio ${ }^{(31)}$. Tais achados se assemelham em parte, no entanto, a escala de sobrecarga (Zarit) também se associou com todos os domínios do WHOQOL-bref e com o IGQV.

Em várias situações os cuidadores tornam-se responsáveis pelos cuidados sem qualquer treinamento prévio e vão aprendendo com a prática cotidiana. Verifica-se que estes carecem de informações e orientações sobre cuidados específicos com o idoso, os quais deveriam ser supridos por profissionais de saúde. Isso pode refletir na sobrecarga evidenciada, muitas vezes, acompanhada de sintomas psicossomáticos. Logo, é reforçada a importância de trabalho específico de prevenção, orientação e tratamento dos cuidadores informais ${ }^{(16)}$. Neste estudo, foi evidenciado inexistência de orientações e informações para cuidadores familiares, por parte dos profissionais de saúde que atuam nas ESF do município em estudo, sobre cuidados aos idosos no domicílio.

Desta forma, devido os enfermeiros que atuam em ESF estarem mais próximos do contexto familiar das pessoas as quais vivem em comunidade, estes profissionais precisam ter sua participação efetiva na promoção da saúde e prevenção de agravos à saúde dos cuidadores de idosos dependentes e independentes, assim como de suas famílias. Certamente, a educação em saúde é considerada estratégia fundamental e eficaz de apoio a essas pessoas.

Isso porque, estar preparado para cuidar vai além da disponibilidade para realizar a tarefa. Portanto, o desafio que se apresenta aos profissionais de saúde, especialmente ao enfermeiro, é o de fornecer adequado apoio ao cuidador no sentido de instrumentalizá-lo para executar o cuidado, além de identificar redes de suporte ${ }^{(25)}$. Neste sentido, torna-se indispensável que o enfermeiro estimule a participação da família e comunidade no cuidado ao idoso e no apoio ao cuidador. Ressalta-se que a autonomia do cuidador-idoso seja preservada nessa decisão de compartilhamento do cuidado.

Além disso, para o enfermeiro prestar cuidado à família do idoso ele precisa refletir sobre sua prática profissional que, muitas vezes, se encontra fundamentada no modelo clínico. E, tratando-se do cuidado a ser desempenhado no domicílio, este exige relação interpessoal satisfatória entre paciente, profissional de saúde, cuidador e família, visto que neste ambiente a valorização da prática baseada nas relações criadas pode estabelecer vínculo indispensável para o cuidado efetivo. Diante da contextualização, evidencia-se que estratégia preventiva como o apoio social é imprescindível para os cuidadores, e o enfermeiro é um dos atores sociais o qual pode contribuir para isso acontecer. Para tanto, estudo ${ }^{(32)}$ aponta a necessidade de capacitação dos profissionais de saúde em habilidades as quais valorizem a pessoa em todo o curso da vida, inclusive quando esta for idosa, eventualmente com alguma incapacidade.

Foi visto que alguns idosos mesmo não sendo dependentes funcionais e/ou cognitivos, sentem a necessidade de algum membro da família ajudá-los no desenvolvimento de suas atividades cotidianas. E, entre os idosos dependentes, verificou-se elevado grau de dependência e com necessidades de cuidados permanentes. Entre os cuidadores, a maior parte é de mulheres, de meia-idade ou idosas, com baixas condições econômicas e presença de doenças.

\section{CONCLUSÕES}

A QV é subjetiva e foi associada a fatores como a sobrecarga de atividades, quantidade de pessoas que residem no mesmo domicílio, idade e renda do cuidador, assim como ao grau de dependência e gênero do idoso. $\mathrm{O}$ apoio social formal é inexistente e o apoio informal ainda acontece 
de maneira incipiente por parte de familiares e/ ou amigos na população estudada. E os cuidadores não tiveram nenhum tipo de orientações/ informações sobre como cuidar do idoso no domicílio. Deste modo, estratégias de fortalecimento do apoio social, orientações sobre o cuidado ao idoso no domicílio, a educação em saúde e o acesso à prática de atividade física, ofertada por meio de políticas públicas de promoção da saúde, se fazem indispensáveis e podem minimizar a sobrecarga de quem cuida, além de favorecer a melhoria da QV do cuidador informal.

Sugere-se a realização de novos estudos, especialmente, de pesquisa interessada junto aos cuidadores familiares de idosos, com a pretensão de evidenciar problemas e possíveis soluções durante a produção e aquisição de conhecimentos. Isso pode favorecer na minimização de fatores associados com a menor percepção de QV desses indivíduos, particularmente, quando os cuidadores também são idosos e apresentam peculiaridades do próprio envelhecimento. Ademais, as práticas de educação em saúde, desenvolvidas por profissionais de saúde, sobretudo, enfermeiros, sobre como prestar o cuidado ao idoso, são necessárias e podem reduzir implicações aos cuidadores (como a sobrecarga) e influenciar positivamente na QV e no desenvolvimento do cuidado consciente e responsável.

Este estudo apresenta limitação por não ter avaliado a variável religião, pois esta tem influenciado no apoio social, assim como não ter aplicado instrumento específico e validado para averiguar de que forma a rede de apoio social contribui na QV dos cuidadores. Acrescido a isso, têm-se as peculiaridades da região, com suas características próprias, como a reduzida escolaridade dos cuidadores, o que tornou algumas questões do WHOQOL-bref complexa quanto à compreensão. Apesar da falta de aplicabilidade do teste piloto para o questionário sociodemográfico e de saúde, acredita-se que não gere comprometimento nos dados, tendo em vista que essas variáveis já são utilizadas nas pesquisas em saúde, conforme estudos apresentados ao longo da discussão. Destarte, acredita-se que em futuros estudos outros instrumentos como os qualitativos, elaborados com linguagem acessível pelos pesquisadores, podem ser utilizados para avaliar a QV em populações como esta.

\section{REFERÊNCIAS}

1. Duarte MGM, Gouveia MAC, Silva AA. Population aging in Brazil: current and future social challenges and consequences. Rev Bras Geriatr Gerontol. 2016; 19(3): 507-19.

2. Onofri Júnior VA, Martins VS, Marin MJS. Atenção à saúde do idoso na Estratégia Saúde da Família e a presença de transtornos mentais comuns. Rev Bras Geriatr Gerontol. 2016; 19(1): 21-33.

3. Reis CCA, Sena ELS, Menezes TMO. Vivências de familiares cuidadores de pessoas idosas hospitalizadas e a experiência de intercorporeidade. Esc Anna Nery. 2016; 20(3): e20160070.

4. Lopes GL, Santos MIPO. Funcionalidade de idosos cadastrados em uma unidade da Estratégia Saúde da Família segundo categorias da Classificação Internacional de Funcionalidade. Rev Bras Geriatr Gerontol. 2015; 18(1): 71-83.

5. Watanabe A, Fukuda M, Suzuki M, Kawaguchi T, Habata T, Akutsu T, et al. Factors Decreasing Caregiver Burden to Allow Patients with Cerebrovascular Disease to Continue in Long-term Home Care. J Stroke Cerebrovasc Dis. 2015; 24(2): 424-30.

6. Mendes CFM, Santos ALS. O cuidado na doença de Alzheimer: as representações sociais dos cuidadores familiares. Saude Soc. 2016; 25(1): 121-32.

7. Tsai Y-H, Lou M-F, Feng T-H, Chu T-L, Chen Y-J, Liu H-E. Mediating effects of burden on quality of life for caregivers of first-time stroke patients discharged from the hospital within one year. BMC Neurology. 2018; 18(50): 1-9.

8. The Whoqol Group. The World Health Organization Quality of Life assessment (WHOQOL): position paper from the World Health 
Organization. Soc Sci Med 1995; 41(10): 1403-9.

9. Day CB, Bierhals CCBK, Santos NO, Mocellin D, Predebon ML, Pizzol FLFD, et al. Nursing home care educational intervention for family caregivers of older adults post stroke (SHARE): study protocol for a randomised trial. Trials. 2018; 19: 96.

10. Moreira ACA, Silva MJ, Darder JJT, Coutinho JFV, Vasconcelos MIO, Marques MB. Efetividade da intervenção educativa no conhecimento-atitude-pratica de cuidadores de idosos. Rev Bras Enferm. 2018; 71(3): 1118-26.

11. Instituto Brasileiro de Geografia e Estatística (IBGE). Censo demográfico 2010. Acesso a informações das cidades. Rio de Janeiro: IBGE; 2010.

12. Programa das Nações Unidas para o Desenvolvimento (PNUD). Atlas do Desenvolvimento Humano no Brasil. Brasília: PNUD; 2013.

13. Lino VTS, Pereira SRM, Camacho LAB, Ribeiro Filho ST, Buksman S. Adaptação transcultural da escala de independência em atividades da vida diária (Escala de Katz). Cad Saude Publica. 2008; 24(1): 103-12.

14. Scazufca M. Brazilian version of the Burden Interview scale for the assessment of burden of care in carers of people with mental illnesses. Rev Bras Psiquiatr. 2002; 24(1): 12-7.

15. Fleck MPA, Louzada S, Xavier M, Chachamovich E, Vieira G, Santos L et al. Aplicação da versão em português do instrumento abreviado de avaliação da qualidade de vida “WHOQOL-bref”. Rev Saúde Pública. 2000; 34(2): 178-83.

16. Gratão ACM, Talmelli LFS, Figueiredo LC, Rosset I, Freitas CP, Rodrigues RAP. Dependência funcional de idosos e a sobrecarga do cuidador. Rev Esc Enferm USP. 2013; 47(1): 137-144.

17. Kahle-Wrobleski K, Andrews JS, Belger M, Ye W, Gauthier S, Rentz DM et al. Dependence levels as interim clinical milestones along the continuum of Alzheimer's Disease: 18-Month Results from the GERAS Obser- vational Study. J Prev Alzheimers Dis. 2017; 4(2): 72-80.

18. Anjos KFA, Santos VC, Teixeira JRB, Boery RNSO, Boery EM, Moreira RM. Calidad de vida de cuidadores familiares de ancianos: una revisión integradora. Rev Cubana Enfermer. 2013; 19(4).

19. Orfila F, Coma-Solé M, Cabanas M, Lombardo FC, Moleras-Seraa A, Pujol-Ribera E. Family caregiver mistreatment of the elderly: prevalence of risk and associated factors. BMC Public Health. 2018; 18: 167.

20. Santos-Orlandi AA, Brito TRP, Ottaviani AC, Rossetti ES, Zazzetta MS, Gratão ACM, et al. Perfil de idosos que cuidam de outros idosos em contexto de alta vulnerabilidade social. Esc Anna Nery. 2017; 21(1): e20170013.

21. Miranda ACC, Sérgio SR, Fonseca GNS, Coelho SMC, Rodrigues JS, Cardoso CL, et al. Avaliação da presença de cuidador familiar de idosos com déficits cognitivo e funcional residentes em Belo Horizonte-MG. Rev Bras Geriatr Gerontol. 2015; 18(1): 141-50.

22. Brigola AG, Luchesi BM, Rossetti ES, Mioshi E, Inouye K, Pavarini SCL. Perfil de saúde de cuidadores familiares de idosos e sua relação com variáveis do cuidado: um estudo no contexto rural. Rev Bras Geriatr Gerontol. 2017; 20(3): 410-22.

23. Polaro SHI, Gonçalves LHT, Nassar SMN, Lopes MMB, Monteiro HK. Dinâmica da família no contexto dos cuidados a adultos na quarta idade. Rev Bras Enferm. 2013; 66(2): 228-33.

24. Silva BMC, Caldas CP, David HMSL, Thiollent MJM. Dificuldades encontradas no cuidado ao idoso com demência: enfrentamento baseado na pesquisa participativa. Rev Bras Geriatr Gerontol. 2018; 21(1): 35-43.

25. Nardi EFR, Sawada NO, Santos JLF. Associação entre a incapacidade funcional do idoso e a sobrecarga do cuidador familiar. Rev Lat Am Enfermagem 2013; 21(5): 1096-103.

26. Costa TF, Costa KNFM, Fernandes MGM, Martins KP, Brito SS. Qualidade de vida de cuidadores de indivíduos com aciden- 
te vascular encefálico: associação com características e sobrecarga. Rev Esc Enferm USP. 2015; 49(2): 0245-52.

27. Bauab JP, Emmel MLG. Mudanças no cotidiano de cuidadores de idosos em processo demencial. Rev Bras Geriatr Gerontol. 2014; 17(2): 339-352.

28. Silva JK, Boery RNSO. O significado de cuidar de uma idosa dependente após o Acidente Vascular Cerebral. Av Enferm. 2017; 35(2): 206-16.

29. Pereira LSM, Soares SM. Fatores que influenciam a qualidade de vida do cuidador familiar do idoso com demência. Cien Saude Colet. 2015; 20(12):3839-51.
30. Coura AS, Nogueira CA, Alves FP, Aragão JS, França ISX, Medeiros KKAS. Quality of life of caregivers of octogenarians: a study using the WHOQOL-Bref. Invest Educ Enferm. 2015; 33(3): 529-38.

31. Wachholz PA, Santos RCC, Wolf LSP. Reconhecendo a sobrecarga e a qualidade de vida de cuidadores familiares de idosos frágeis. Rev Bras Geriatr Gerontol. 2013; 16(3): 51326.

32. Giacomin KC, Firmo JOA. Velhice, incapacidade e cuidado na saúde pública. Cien Saude Colet. 2015; 20(12): 3631-40. 